\title{
Novel kinematic indices for quantifying upper limb ability and dexterity after cervical spinal cord injury
}

\author{
Ana de los Reyes-Guzmán · Iris Dimbwadyo-Terrer • Soraya Pérez-Nombela • \\ Félix Monasterio-Huelin · Diego Torricelli · José Luis Pons · Angel Gil-Agudo
}

\begin{abstract}
Loss of motor function is a consequence after cervical spinal cord injury. Three-dimensional kinematic analysis equipments are used for quantifying human movements in clinical laboratories. These systems may provide objectivity to the patient assessments. Nowadays, the kinematic variables found in the literature have some deficiencies, and the efficient management of these data sets is a demand and a challenge in the clinical setting. The aim of the present paper is to propose a set of novel kinematic indices, as a combination of kinematic variables, for quantifying upper limb motor disorders in terms of characteristics in relation to ability and dexterity such as accuracy, efficiency, and coordination. These indices are defined for measuring patients' motor performance during the activity of daily living of drinking from a glass. This task is included within the upper limb rehabilitative process that patients receive. The main contribution of this research, with the aim of detecting upper limb impairments in patients, consists of the proposal of three kinematic indices
\end{abstract}

from experimental data, whose results are dimensionless and relative to a pattern of healthy subjects. We hope that kinematic indices proposed are a step toward the standardization of the quantitative assessment of movement characteristics and functional impairments.

Keywords Kinematic indices - Functional assessment . Upper limb · Activities of daily living · Spinal cord injury . Neurological diseases
Abbreviations
SCI Spinal cord injury
ADL Activity of daily living
UL Upper limb

\section{Introduction}

Loss of motor function is a hallmark consequence after spinal cord injury (SCI). The incidence of SCI varies greatly worldwide from 12.1 to 57.8 SCI cases per million depending on the countries [39]. Among them, the upper limb (UL) is affected in more than $50 \%$ of cases [44]. UL strength is impaired to some extent in people who have suffered cervical SCI, making it difficult for them to perform many activities of daily living (ADL) essential for their autonomy. Therefore, these patients experience sharp limitations in their level of activity and participation in the social setting, as people who have suffered another central nervous system injury, such as stroke [4].

In this context, quantitative measures of human movement quality are significant in the rehabilitation field for expressing the outcomes during rehabilitation treatments, discriminating between healthy and pathological conditions [45], and for helping in the decision making in the clinical setting. 
The UL function assessment is mainly performed based on clinical scales. Some of them assess the level of dependence or independence in performing ADL, such as the Barthel Index [26] and the Functional Independence Measure (FIM) scale [19]. Other scales, such as the Jebsen-Taylor Hand Function [17], measure UL function in terms of the skill and ability to perform specific functional tasks. These scales are general, so can be applied to different populations of subjects with different pathologies. However, there are other scales developed for a specific injury, in order to make the functional assessments more sensitive to changes. An example is the Spinal Cord Independence Measure (SCIM) [7], specific for assessing SCI. Tuijl proposed a review of clinical scales, specific for SCI, classified into strength, ADL and functional scales [41].

These clinical scales are standardized measure instruments, valid for their use in the clinical setting because they have been previously validated in large patients samples. However, although these scales are easy to administer, the main disadvantage of these quantitative measures is that they have a high subjective component, depending on the observer who visually scores the test. Another aspect to take into account is the sensitivity of the scales mainly to gross changes in the health status or UL function, so that subtle changes in the subject may not be detected. Moreover, they sometimes present saturations in the scoring system, and as consequence, improvements which may occur above a certain threshold are not detected. For these reasons, it seems that only clinical scales are not enough, by themselves, to assess motor strategies used during movements performance [6]. So, it's necessary to find measure instruments which, in combination with clinical scales, provide objectivity, and at the same time, solve the limitations that scales have.

Biomechanical studies are examples of objective methods, in which several technologies can be used to collect data from the subjects [46]. One kind of these studies are the kinematic analysis that provides objective information about motor strategies associated with UL goal-oriented tasks. Data collected must be necessarily treated and reduced to a set of variables that, a priori, have a simpler interpretation for their use in the clinical practice.

Recently, in our previously work, an overview was published in relation to objective kinematic variables found in the literature to quantify the UL function regardless the system used for extracting kinematic data and the UL movements analyzed. The variables and metrics found were described and classified according to the movement characteristic that they quantified [13]. However, some deficiencies have been detected in these metrics. They usually consist of the kinematic variables directly obtained from movement analysis equipments, expressed as absolute units, and sometimes, an increase in the measured variable corresponds to a decrease in the movement characteristic that represents [10, 36], producing an inconsistency in the outcomes interpretation. Moreover, the most frequently analyzed movement is the reaching movement $[6,8-10,18$, $23,29,36]$. Studies in complete ADL have been performed mostly in healthy subjects $[1,25,30,31,35,38]$. Some studies have been addressed in stroke [20, 22, 32-34] and cerebral palsy $[5,16,21]$ populations, being very scarce in SCI populations [14].

These deficiencies, detected in clinical scales and the kinematic variables found in the revision performed, motivated this research. The objective of the present paper is to define and to develop three novel objective kinematic indices related to the UL dexterity and ability, such as accuracy, efficiency, and coordination, and to analyze their discriminative capability for detecting UL functional impairments in people who have suffered cervical SCI.

\section{Methods}

\subsection{Participants}

A total of 18 people divided into three groups participated in the study: a healthy group $(n=7)$; and two groups of patients with motor complete cervical SCI with metameric level C6 $(n=7)$ and C7 $(n=4)$. This patients' sample was chosen because the two groups have UL functional differences between them. Hence, patients that were included into the $\mathrm{C} 6$ group are more affected that those in the other one. $\mathrm{C} 6$ patients retain control of the elbow flexion and the wrist extensor muscles but lose active extension of the elbow, whereas $\mathrm{C} 7$ patients retain active control of all these muscles. However, both patients groups lose the ability to perform active prehension movements [27]. All participants were right-handed. Background data of participants are provided in Table 1 . The patients screened had to fulfill the following criteria to be included in the study: age 16-65 years, injury of at least 6-month duration and level of cervical injury, C6 or C7, classified according to the American Spinal Injury Association (ASIA) [28] scale into grades A or B. Patients who presented any vertebral deformity, joint constraint, surgery or any of the UL, balance disorders, dysmetria due to associated neurologic disorders, visual acuity defects, cognitive deficit, or head injury associated with the SCI were excluded. Patients were classified into C6 and C7 SCI by a physical examination. The UL Motor Index was obtained [28], with the assessment of the strength of five muscles groups of the right UL by a physiotherapist. Each muscle group can be assessed between 0 (no function) to 5 (normal function) with a total of 25 points. The guidelines of the Declaration of Helsinki were followed in every case. Informed consent 
Table 1 Demographic and functional characteristics of the sample analyzed $(n=18)$

\begin{tabular}{llcr}
\hline Variables & $\begin{array}{l}\text { Healthy subjects } \\
(n=7)\end{array}$ & $\begin{array}{l}\text { C6 SCI } \\
(n=7)\end{array}$ & \multicolumn{1}{c}{$\begin{array}{l}\text { C7 SCI } \\
(n=4)\end{array}$} \\
\hline Sex (Male) & $3(42.8)$ & $4(57.4)$ & $4(100)$ \\
Age $\left(\right.$ years) $^{\mathrm{b}}$ & $28.0(5.0)$ & $34.0(5.0)$ & $30.5(10.0)$ \\
Height $(\mathrm{cm})^{\mathrm{b}}$ & $168.0(20.0)$ & $175.0(10.0)$ & $184.0(10.0)$ \\
Weight $(\mathrm{Kg})^{\mathrm{b}}$ & $65.0(21.1)$ & $90.2(7.1)$ & $79.0(9.1)$ \\
Months since injury $^{\mathrm{b}}$ & - & $8.5(2.2)$ & $7.5(1.8)$ \\
ASIA (grade A) $^{\mathrm{a}}$ & - & $3(42.8)$ & $2(50)$ \\
ASIA (grade B) $^{\mathrm{a}}$ & - & $4(57.2)$ & $2(50)$ \\
Motor Index $^{\mathrm{b}}$ & $25.0(0.0)$ & $13.0(3.0)$ & $14.5(2.0)$ \\
\hline
\end{tabular}

${ }^{a}$ Frequency and percentage for categorical variables

b Mean and standard deviation for continuous variables

was obtained from all individual participants included in the study, which was approved by the Local Ethics Committee, Toledo, Spain.

\subsection{Procedure}

UL movement was recorded by using the Codamotion system (Charnwood Dynamics, Ltd, UK) based on active markers. A total of 18 markers were used, placed on the skin surface in the trunk and the right arm (Fig. 1). 3D marker positions are calculated instantly with a spatial resolution of $0.1 \mathrm{~mm}$. All the participants, instrumented with Codamotion markers and seated in a wheelchair in front of a table, performed only one experimental session with five repetitions of the $\mathrm{ADL}$ of drinking. All this experimental setup was described in detail in a previous study [14]. The only difference within the experimental protocol was the distance to the glass placed on the table. In this study, the glass was placed in the midline of the body, to the $75 \%$ of the maximal UL reaching [5] with the aim of minimizing compensatory movements.

The drinking task included reaching and grasping the glass, lifting the glass to the mouth, drinking a swallow, releasing the glass on the table, and returning to the starting position. All the participants performed the movement with the right arm. They were instructed for initiating the drinking task at a comfortable self-selected speed. Five trials of the task were recorded for processing.

For computing joint kinematics and hand trajectories from position data of Codamotion markers, a biomechanical model previously published was used [14]. Data were filtered with second-order Butterworth filter with a cutoff frequency of $4 \mathrm{~Hz}$. The model included the trunk, right arm, right forearm, and the right-hand body segments. The body segments are considered as rigid solids joined between them by rotation centers estimated by Codamotion markers

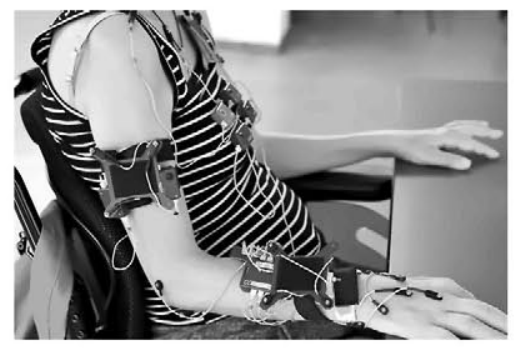

Fig. 1 A patient instrumented with Codamotion markers during an experimental session

placed on bony prominences on the skin surface. The six model degrees of freedom (DoF) were placed divided into joints: three in the shoulder joint (flexion-extension, abduction-adduction and external-internal rotation movements); two in the elbow (flexion-extension and pronation-supination movements); and one in the wrist joint (flexion-extension movement). All the DoF were validated by the performance of analytical movements in which only one joint was involved.

To facilitate analysis, the drinking ADL was broken down into five consecutive phases, following Murphy's study guidelines [31]: reaching (included grasping the glass), forward transport, drinking, distal transport (included releasing the object), and returning to the starting point.

The new contribution of this research was based on exporting kinematic data to MATLAB (The MathWorksInc) software for calculating the kinematic indices proposed during the complete cycle of the drinking task.

\subsection{Kinematic indices}

Kinematic indices assess UL ability and dexterity, such as accuracy, efficiency, and coordination. Motor learning is fundamental to neurological rehabilitation. UL functional deficits after neurological diseases are reflected in compensatory movements in proximal joints. Hence, these indices were designed to detect UL functional impairments.

Accuracy and efficiency indices were defined from 3D position data of the hand during the movement. However, the coordination index was defined from the range of motion of shoulder and elbow joints.

\subsubsection{Accuracy and efficiency indices}

The accuracy and efficiency indices were assessed from the hand trajectory during the ADL movement. The hand trajectory, $\mathrm{s}[n]$, was considered as the module of the hand position vector.

$s[n]=\sqrt{x[n]^{2}+y[n]^{2}+z[n]^{2}}$ 


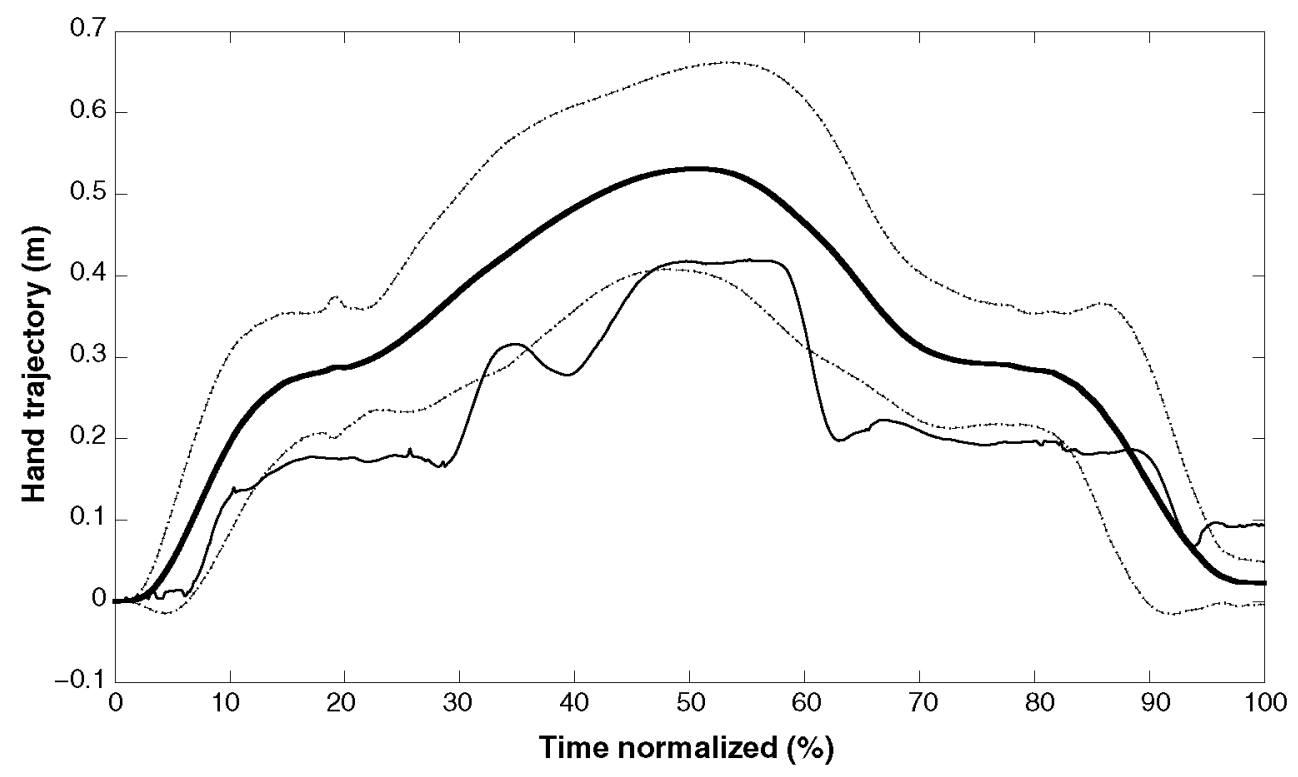

Fig. 2 Hand trajectories during a complete cycle of the drinking ADL. Gross continuous line represents the reference trajectory for the healthy pattern. Discontinuous line represents the standard deviation

The mean trajectory of the hand movement for the pattern of healthy subjects, considered the reference trajectory, and the real trajectory for a patient with cervical SCI are shown in Fig. 2. For comparisons between trajectories, they were interpolated to a duration equal to $N=5000$ samples. To the sample frequency of Codamotion equipment, this sample size corresponded to $25 \mathrm{~s}$ of movement. This time was enough for patients could execute a complete cycle of the ADL of drinking.

Accuracy index The accuracy index was computed as the product of three terms: $\alpha, \rho$, and BN. $\alpha$ is a dependent function on the computed mean distance between the reference trajectory and the real trajectory performed by the patient; $\rho$ is a dependent function on the shape and trend of both trajectories; and $\mathrm{BN}$ is a parameter that represents the percentage of the drinking task in which the hand trajectory performed by the subject is within the dispersion band considered acceptable around the reference trajectory.

The accuracy index, A, was computed from Eq. 2. Posteriorly, the result was relativized as the mean accuracy value for the pattern of healthy subjects, $P_{\text {ref. }}$.

$$
\begin{array}{r}
A=\alpha \cdot \rho \cdot \mathrm{BN} \\
A_{\text {norm }}=\frac{A}{A_{\text {ref }}} \cdot 100
\end{array}
$$

- The mean absolute value of the distance, $d_{m}(\mathrm{~cm})$, from each point of both time normalized trajectories (the reference trajectory and the real one performed by around the mean trajectory. The thick continuous line represents the real trajectory performed by a SCI patient

the patient), was obtained. $\alpha$ was defined as a sigmoid function (Eq. 3; Fig. 3). The value was between 0 and 1 depending on the mean distance value, $d_{m}$. Decrease values for $\alpha$ reflect increasing mean distances between trajectories. The $\beta_{1}$ constant was fixed to 20 because this value maintains the sigmoid curve shape and the decreasing is less abrupt than for greater values.

$\alpha=\alpha_{\min }+\left(1-\alpha_{\min }\right) \frac{1}{1+e^{\beta_{1}\left(d_{m}-\theta\right)}}$

$\alpha_{\min }$ was saturated $\left(\alpha_{\min }=0.25\right)$ to avoid obtaining zero points in accuracy index.

- $\rho$ was computed by the Pearson coefficient between both trajectories, the real and the mean trajectory for healthy pattern. So this parameter can vary between 0 and 1 .

- The third component for calculating the accuracy index was BN. BN was defined as the percentage of the drinking cycle that the real trajectory performed by the subject was within the dispersion band considered acceptable. So, several band widths were analyzed as $\mathrm{n}$ times the standard deviation around the mean trajectory. The choice of the dispersion band width is explained in the results section.

Efficiency index Efficiency index, $E$, was obtained by computing the path length, $l_{\text {path }}$, of the hand trajectory performed by the patient, normalized by the mean hand 
Fig. $3 \alpha$ parameter values as a function of the mean distance between the real trajectory performed by a patient and the reference trajectory. Several values for the $\beta_{1}$ constant have been analyzed

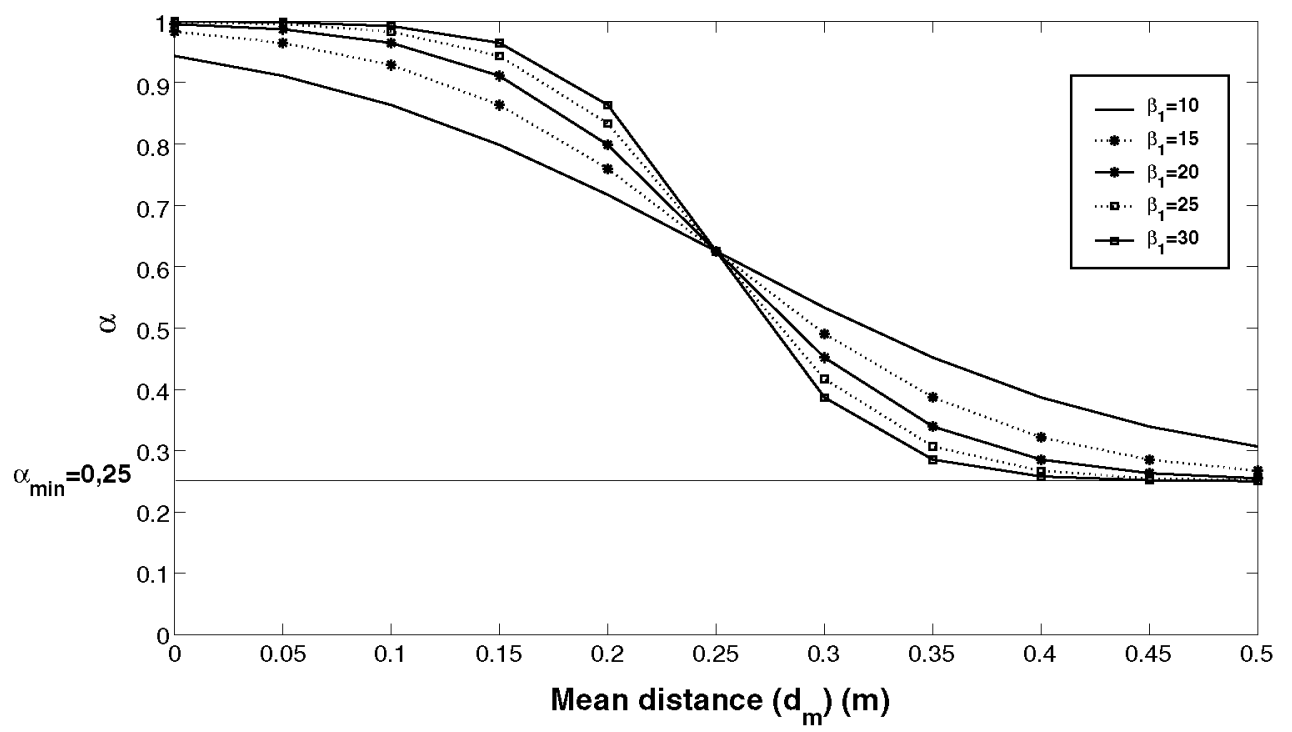

path length corresponding to the healthy pattern, $l_{\text {path_ref }}$. The result was inverted (Eq. 4) because normally, patients perform movements with longer trajectories than healthy people due to movement deviations around the desired trajectories [10]. Path lengths were obtained by summing the distances between two consecutive points of the trajectories. In this index, decreasing values in the real path length performed by a patient reflect an improvement of the efficiency index during the drinking task.

$E=\frac{l_{\text {path_ref }}}{l_{\text {path }}} \cdot 100$

\subsubsection{Coordination index}

In the literature, this index has been always applied to the reaching movement $[15,31,32,43]$. However, in this research, the coordination index proposed, $C$, was designed for assessing the simultaneous action of shoulder and elbow joints during a complete ADL. It was calculated from the Pearson coefficient for each phase that implied a hand displacement: reaching (included grasping), forward and distal transport and returning to the starting position. For reaching and returning phases, the Pearson coefficients, $\rho_{\text {rea }}$ and $\rho_{\text {ret }}$, were calculated between shoulder flexion and elbow extension. While for forward and distal transport, the correlation indices, $\rho_{\mathrm{ft}}$ and $\rho_{\mathrm{dt}}$, were computed between shoulder abduction and elbow extension.

Finally, the coordination index was computed as a weighted average from the computed correlation indices (Eq. 5). The duration of the complete ADL, $N_{\mathrm{ADL}}$, and each drinking ADL phase was taken into account: $N_{\text {rea }}$ and $N_{\text {ret }}$, samples during the reaching and returning phases; $N_{\mathrm{ft}}$ and $N_{\mathrm{dt}}$, samples during the forward and distal transport phases. Then, the index was normalized by the mean coordination for the pattern of healthy subjects, $C_{\text {ref }}(\mathrm{Eq} .6)$.

$C=\frac{\rho_{\text {rea }} \cdot N_{\text {rea }}+\rho_{\mathrm{ft}} \cdot N_{\mathrm{ft}}+\rho_{\mathrm{dt}} \cdot N_{\mathrm{dt}}+\rho_{\text {ret }} \cdot N_{\text {ret }}}{N_{\mathrm{ADL}}}$

$C_{\text {norm }}=\frac{C}{C_{\text {ref }}} \cdot 100$

\subsection{Statistical analysis}

Statistical analysis was performed with SPSS (Statistical Packages for the Social Sciences, release 12.0 for Windows, SPSS Inc, Chicago, IL). In the analysis of kinematic indices, the mean value of the five recordings was used.

A descriptive analysis was made of the clinical and functional variables by calculating the median and interquartile range of the quantitative variable and the frequencies and percentages of the qualitative variables.

For accuracy and coordination indices computing, the Pearson correlation index was applied.

To check the discriminative capability of kinematic indices proposed, comparisons between healthy and SCI patients, and between patients with different severity level were made. The Kruskal-Wallis test was applied to find possible differences in each variable between the three groups analyzed; the Kruskal-Wallis test is $p<0.05$, the equivalence of behavior between groups can be rejected, and a pairwise comparison can be made using the Mann-Whitney test. The Bonferroni correction was applied, which takes into account randomness due to multiple comparisons.

The repeatability of the experimental protocol during the ADL of drinking was analyzed in our previous study [14]. 


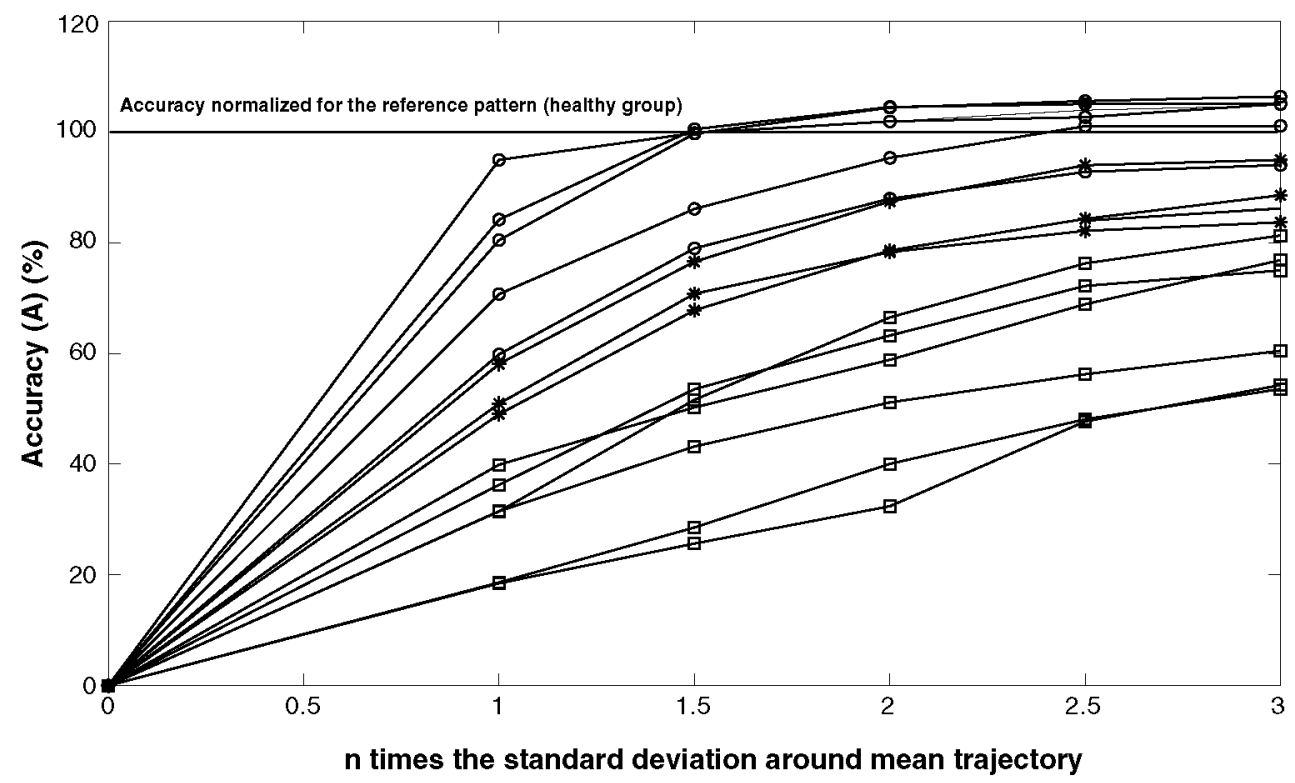

Fig. 4 Accuracy index values based on different widths of the dispersion band. A circle represents the accuracy index for healthy people, a square represents the accuracy index for $C 6 \mathrm{SCI}$ people, and the

asterisk is used for $C 7 \mathrm{SCI}$ people. Abscises axis represents the width of the dispersion band around the reference trajectory

\section{Results}

The sample analyzed was divided into three groups that were matched in age, weight, and height. So, no statistical significant differences were found for demographic characteristics among the three groups.

\subsection{Choosing the width of the band dispersion for accuracy index computing}

For computing BN parameter, the width of the dispersion band was chosen. The influence of the dispersion band size to the accuracy index was analyzed (Fig. 4) in the three populations analyzed. Accuracy was computing for each dispersion band size, with fixed $\alpha$ and $\rho$ parameters within each subject.

From this analysis, firstly, a narrow dispersion band (1 and 1.5 times the standard deviation around the reference trajectory corresponding to the healthy mean trajectory) was considered. For both deviation values, the results of the accuracy index were low in the three groups analyzed, including within the healthy group, due to a very low value of the BN parameter. As a consequence, in both cases, the variability within the healthy group is greater too. However, with a wide dispersion band, the Accuracy index was very high for patients, and the results were saturated by the healthy subjects ( 2.5 and 3 times the standard deviation around the reference trajectory) (Fig. 4). So, the best choice seemed to be a compromise between a narrow and

a wide dispersion band, corresponding to a dispersion band of twice the standard deviation around the mean value.

\subsection{Accuracy and efficiency indices}

These indices were computed from hand kinematic data. The Accuracy index detected differences between the three groups analyzed. Accuracy was greater in healthy subjects than in C6 and C7 SCI groups ( $p<0.01$ and $p<0.05$, respectively) during the complete $\mathrm{ADL}$. Moreover, this index discriminated between cervical injury levels $\mathrm{C} 6$ and C7 $(p<0.05)$. The C6 SCI group was functionally more affected than $\mathrm{C} 7$ group, and the accuracy result is lower in C6 than in C7 SCI group (Table 2). For this index, the box plots corresponding to the three groups weren't overlapped between them (Fig. 5a), showing the discriminative capacity of the accuracy index.

The mean distance between trajectories was $3.73 \mathrm{~cm}$ in healthy subjects, $8.86 \mathrm{~cm}$ in C6 SCI group, and $4.99 \mathrm{~cm}$ in C7 SCI group. The standard deviation in SCI people is greater than in healthy group. So, $\alpha$ parameter is greater in C6 SCI patients than in healthy $(p<0.05)$ and C7 SCI groups $(p<0.01$; Table 2 ). The same behavior was appreciated in relation to the $\rho$ parameter, which compares the curve shape between the real and reference trajectory performed by the patient and the healthy pattern, respectively. The BN parameter was able to detect differences between healthy and pathological conditions $(p<0.05$ and $p<0.01$ in C7 and C6 SCI groups, respectively). So, the real trajectories performed 
Table 2 Accuracy and efficiency kinematic indices for each group analyzed

\begin{tabular}{llll}
\hline $\begin{array}{l}\text { Kinematic } \\
\text { variables }\end{array}$ & $\begin{array}{l}\text { Healthy subjects } \\
(n=7)\end{array}$ & $\begin{array}{l}\text { C6 SCI } \\
(n=7)\end{array}$ & $\begin{array}{l}\text { C7 SCI } \\
(n=4)\end{array}$ \\
\hline $\begin{array}{l}\text { Accuracy index } \\
(\% \text { pattern })\end{array}$ & $104.22(6.29)^{\mathrm{a}, \mathrm{c}}$ & $70.79(19.49)^{\mathrm{b}, \mathrm{c}}$ & $90.13(6.89)^{\mathrm{a}, \mathrm{b}}$ \\
Alfa $(0-1)$ & $0.98(0.00)^{\mathrm{a}}$ & $0.94(0.04)^{\mathrm{a}, \mathrm{c}}$ & $0.98(0.00)^{\mathrm{c}}$ \\
Rho $(0-1)$ & $0.97(0.02)^{\mathrm{a}}$ & $0.84(0.08)^{\mathrm{a}, \mathrm{c}}$ & $0.94(0.27)^{\mathrm{c}}$ \\
BN $(0-100)$ & $95.92(4.23)^{\mathrm{a}, \mathrm{c}}$ & $65.69(16.79)^{\mathrm{c}}$ & $87.48(4.48)^{\mathrm{a}}$ \\
$d_{\text {max }}(\mathrm{cm})$ & $10.37(2.77)^{\mathrm{a}}$ & $21.35(5.52)^{\mathrm{a}, \mathrm{c}}$ & $14.18(3.25)^{\mathrm{c}}$ \\
$d_{m}(\mathrm{~cm})$ & $3.73(1.29)^{\mathrm{a}}$ & $8.86(3.63)^{\mathrm{a}, \mathrm{c}}$ & $4.99(6.89)^{\mathrm{c}}$ \\
Efficiency index & $100.52(5.83)^{\mathrm{a}}$ & $99.29(10.45)^{\mathrm{a}}$ & $97.75(6.72)$ \\
$(\%$ pattern $)$ & & & \\
\hline
\end{tabular}

Results are expressed as the median and interquartile range

a,b $(p<0.05)$ and ${ }^{\mathrm{c}}(p<0.01)$

by C6 SCI patients were within the dispersion band around the reference trajectory during a $65.69 \%$ of the movement cycle. However, this value was the $87.48 \%$ during the movement cycle for the C7 SCI group.

The efficiency index was defined from the hand path length. Patients performed the drinking ADL with a trajectory longer than healthy people, resulting in a lower Efficiency index. Although the median value for this index is greater in C6 SCI people than in C7 SCI group, the dispersion within the group is greater too and both box plots were overlapped (Fig. 5b). So, statistically significant differences were found between healthy and C6 SCI people ( $p<0.05$; Table 2$)$.

\subsection{Coordination index}

In the three study groups, there was a strong coordination between the shoulder and elbow joint angles in the
Table 3 Coordination index for each group analyzed

\begin{tabular}{llll}
\hline $\begin{array}{l}\text { Kinematic } \\
\text { variables }\end{array}$ & $\begin{array}{l}\text { Healthy subjects } \\
(n=7)\end{array}$ & $\begin{array}{l}\text { C6 SCI } \\
(n=7)\end{array}$ & $\begin{array}{l}\text { C7 SCI } \\
(n=4)\end{array}$ \\
\hline $\begin{array}{l}\text { Coordination } \\
(\% \text { pattern })\end{array}$ & $115.31(7.28)^{\mathrm{a}}$ & $92.85(8.88)^{\mathrm{a}}$ & $102.12(13.47)$ \\
$C_{\text {rea }}($ Pearson \%) & $87.88(5.91)$ & $87.13(12.86)$ & $74.78(24.27)$ \\
$C_{\mathrm{ft}}($ Pearson \%) & $96.66(1.90)^{\mathrm{a}, \mathrm{b}}$ & $65.17(24.79)^{\mathrm{a}}$ & $87.18(6.33)^{\mathrm{b}}$ \\
$C_{\mathrm{dt}}($ Pearson \%) & $88.03(12.39)$ & $73.93(18.55)$ & $79.42(14.16)$ \\
$C_{\text {ret }}($ Pearson \%) & $84.66(20.02)$ & $82.85(25.97)$ & $74.66(29.18)$ \\
Coordination & $90.75(9.69)^{\mathrm{a}}$ & $72.63(6.42)^{\mathrm{a}}$ & $82.92(12.65)$ \\
$(0-100)$ & & & \\
\hline
\end{tabular}

Results are expressed as the median and interquartile range

a $(p<0.05)$

${ }^{\mathrm{b}}(p<0.01)$

flexion-extension movements (Table 3). The correlation index, expressed as a percentage, was high in the three groups analyzed during the ADL of drinking (up $72.63 \%$ in the C6 SCI group to $90.75 \%$ in healthy subjects). However, significant differences were found between healthy subjects and C6 SCI patients $(p<0.05)$, in which the box plots weren't overlapped (Fig. 6). The most important differences were observed during the forward transport phase within the ADL of drinking. In this movement phase, the correlation index was moderated $(65.17 \pm 24.79 \%)$ in the C6 group, in contrast to a very high correlation in healthy people $(96.66 \pm 1.90 \%)(p<0.05)$. These differences were obtained between C7 SCI patients and healthy people too $(p<0.01$; Table 3$)$.

Within the reaching, distal transport and returning to the starting point phases, significant differences were not found between the three populations analyzed.

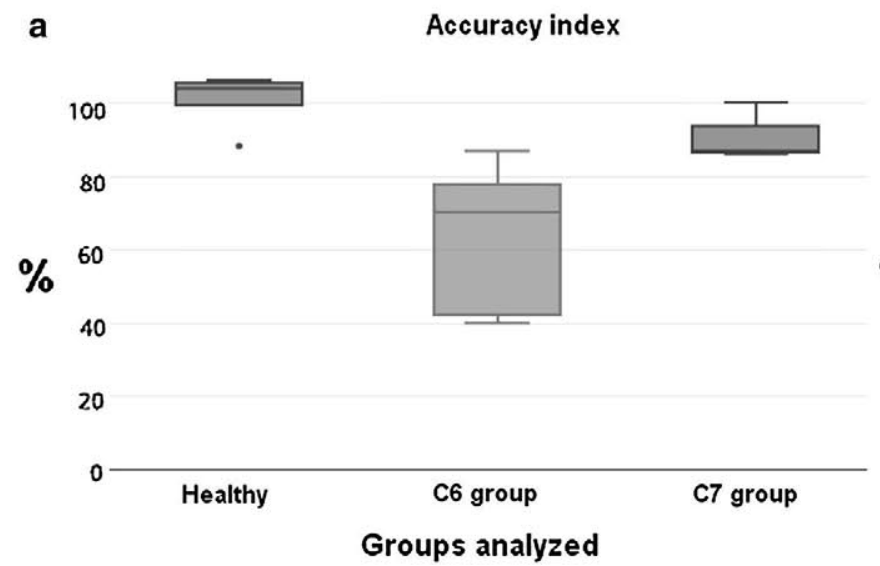

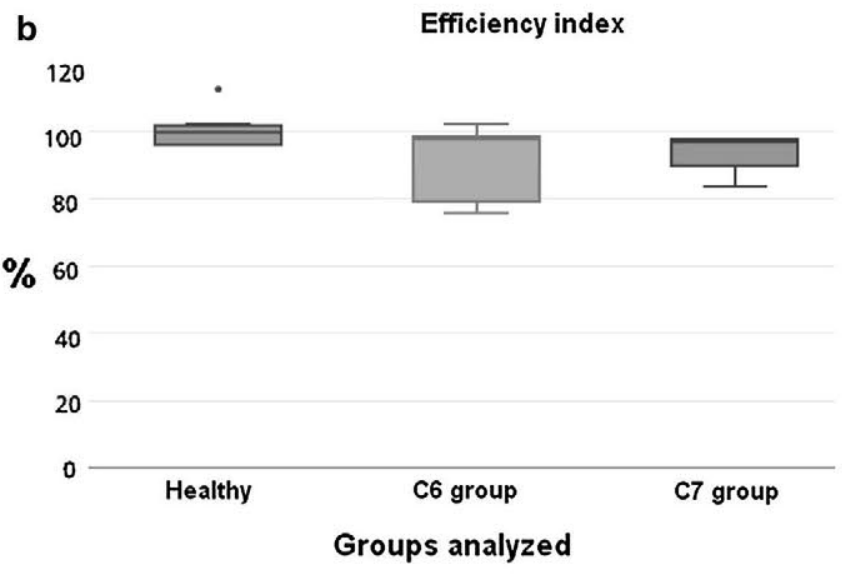

Fig. 5 Box plots for the three groups analyzed for the accuracy (a) and efficiency (b) indices. Each box plot represents the median, the amplitude of the first and third quartile and the maximum and minimum values 
Fig. 6 Box plots for the three groups analyzed for the coordination index. Each box plot represents the median, the amplitude of the first and third quartile and the maximum and minimum values
Coordination index

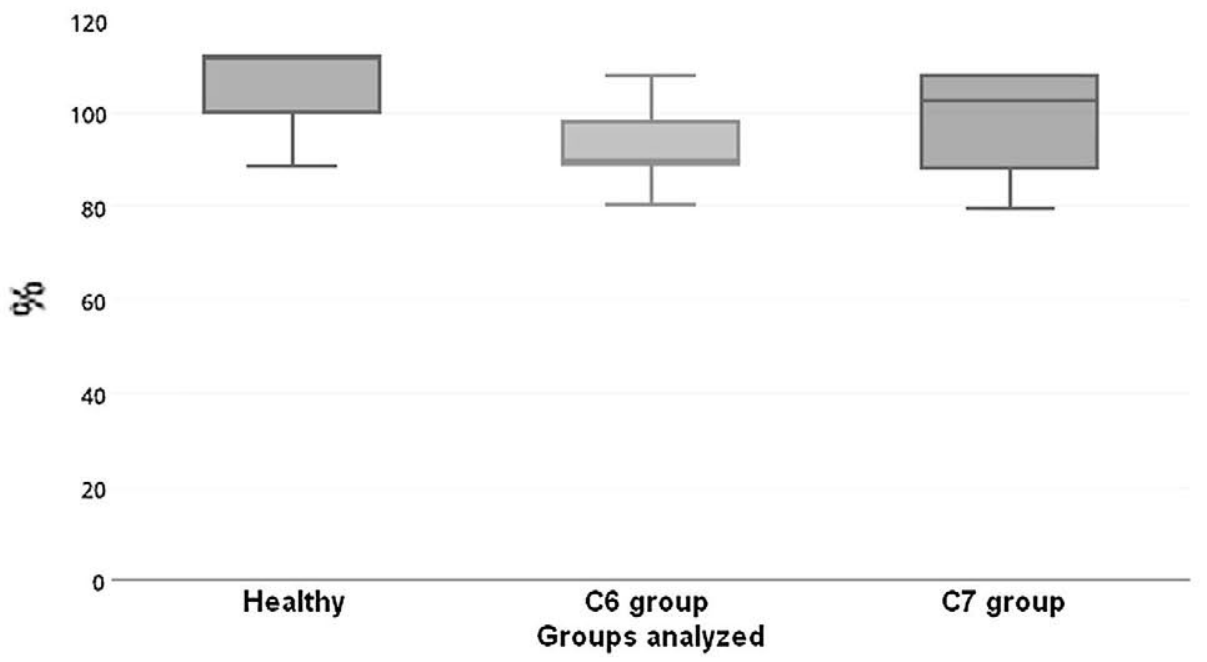

\section{Discussion}

In this paper, three novel kinematic indices for quantifying movement accuracy, efficiency and coordination, respectively, during the performance of the $\mathrm{ADL}$ of drinking have been defined and computed by means of a mathematical formulation. The results suggest that these indices are discriminative in terms of UL functionality between the populations analyzed.

\subsection{Kinematic indices proposed}

The kinematic indices presented in this research have been computed from the hand path during the performance of the drinking ADL, as for example, accuracy and efficiency indices. However, the coordination index has been computed from shoulder and elbow joint movements. This set of indices allows the assessment of these motor control aspects, providing to the clinical staff useful information in relation to the UL performance.

The accuracy index is a measure of the movement quality in terms of deviation of a subject's movement from a theoretical or desired trajectory [10]. This index has been considered a measure of the error in accuracy [10], and in the literature, it was also called as "movement deviation" or "tracking error" [42]. In the reviewed articles, this index has been computed as the mean distance from the Euclidean distance between points of the real and theoretical hand trajectories $[8,10,11]$. Applied to reaching movements, the theoretical trajectory has been the straight line between starting and ending points $[5,6,12,18,22$, $29,40]$. However, in this research, the accuracy index proposed takes into account a reference trajectory equal to the mean of all trajectories performed by a healthy subjects group. The mean distances measured between the real and the reference trajectory are greater in patients groups $(88.6 \pm 36.30 \mathrm{~mm}$ in C6 SCI and $49.90 \pm 68.90 \mathrm{~mm}$ in C7 SCI patients) than in healthy subjects $(37.30 \pm 12.90 \mathrm{~mm})$. These mean distances are greater than those measured by Colombo [10], in which the mean distance values obtained were between $20.98 \pm 15.53$ and $32.94 \pm 13.51 \mathrm{~mm}$. It is necessary to take into account that, in this research, the movement analyzed corresponds to the complete drinking ADL, a more complex movement that the movement analyzed by Colombo [10].

However, similar studies haven't been found in the literature, in relation to a global accuracy index like the one proposed in this research. Each parameter within the accuracy index has detected some differences between healthy and pathological populations, so the global index is able to discriminate between the three groups analyzed. In presence of less functional impairments (C7 SCI against C6 SCI patients), the hand trajectory is more adapted to the healthy pattern.

Moreover, the spatial deviation, the time variability of the trajectory, was analyzed in the literature [37]. In this research context, this topic has been analyzed in the efficiency index. This index was computed from the hand trajectory length as the Euclidean distance between consecutive samples. Then, the result was normalized by the mean length for the healthy pattern. This index was found in the literature applied to the reaching movement $[6,18,22,29$, 40]. Trajectory length values were not found in the literature. Instead of that, only the ratio between the length of the real and theoretical trajectory was reported [10]. So, values greater than one correspond to less efficient movements due to the execution of longer trajectories [6, 40]. Because of this, in this research, the efficiency index has been inverted for greater results correspond to well-executed movements in terms of efficiency. 
In relation to the coordination index, it was found in the literature applied to the reaching movement and computed from the flexion-extension movements of the shoulder and elbow joints [15, 31, 32, 43]. Other study computed the movement coordination during the forward transport phase within the ADL of drinking, from the flexion-extension movement in elbow joint and abduction-adduction movement in shoulder joint [24]. However, in this research, the coordination index has analyzed the complete ADL of drinking, as a weighted average from the correlation analysis of each ADL phase with a hand displacement: reaching, forward and distal transport and returning to the starting point. Although the coordination was high in the three groups analyzed, this index was statistically different between C6 SCI patients and the other two groups. Murphy analyzed the movement coordination for the reaching phase within the ADL of drinking, applied to healthy and stroke people, obtaining the lowest results in moderate stroke people [32].

The results show that the proposed indices are adequate for detecting functional UL impairments, when they have been applied to people with different level of motor complete cervical SCI.

\subsection{Contributions of the kinematic indices}

The contributions of the kinematic indices developed are presented following the order shown in the introduction section in relation to the deficiencies found in the kinematic indices reviewed in the literature [13].

The kinematic metrics found in the literature usually consist of the kinematic variables directly obtained from movement analysis equipments, expressed as absolute values in the corresponding units depending on the kinematic variable analyzed $[9,14,31,36]$. The kinematic indices defined in this research are novel by proposing a mathematical formulation for each one as a combination of kinematic variables and expressing the results in a dimensionless format and relative to a reference pattern of healthy subjects. To present the indices results relative to a reference pattern allows to solve the ceiling effect observed in some clinical scales [41] and to offer the possibility of a subject overcomes the scoring of the reference pattern in the kinematic indices proposed. On the other hand, other advantage of this scoring system proposed is that may allow an easier understanding of the patient progress with respect to himself and to what extent his motor performance gets closer or away with respect to the reference pattern.

In this regard, there may be an inconsistency in the kinematic metrics due to an increase in the variable corresponds to a decrease in the movement characteristic that the index represents. For example, it occurs for the smoothness index expressed as the peaks number in the velocity profile during the movement [36]. This effect has been found in the literature, in the movement efficiency index computed as the ratio between the hand path length performed by the patient and a theoretical path length considered the straight line between the initial and ending point. This formulation has been applied to reaching movements, considering the theoretical path length the straight line between the initial and ending points [13]. From this computation, a ratio near to one indicates a good motor performance. However, the higher this ratio, greater the deviation between both trajectories (the ideal and real trajectories), and therefore, worst motor performance [10]. So in this research, this problem has been solved, and all indices have been designed following a scoring system, chosen for that an increase in their scoring means a better functional state of the patient.

Other important contribution of this research is the UL assessment during the performance of complete ADL, while the most research articles are centered in the reaching and point-to-point movement analysis [6, 8-10, 18, 23, $29,36]$. The kinematic studies for analyzing UL movement during the execution of ADL have been performed mostly in healthy people $[1,25,30,31,35,38]$, being scarce in SCI populations [14]. In this research, the ADL of drinking from a glass has been selected as a representative ADL because its correct execution for maintaining the glass in the hand requires UL control, coordination, accuracy, and force. These characteristics and motor control aspects are impaired in presence of neurological diseases. In a clinical point of view, the understanding in relation to the UL functional deficits during the execution of ADL is very interesting taking into account the rehabilitation objectives, centered in reaching the maximal independence level of patients in ADL execution. For that reason, although the kinematic indices have been applied to patients with cervical SCI, this methodology could be applied to people with other neurological pathologies that produce UL movement disorders such as stroke or cerebral palsy.

\subsection{Study limitations}

This study has some limitations. Firstly, in relation to the size of the sample analyzed, the number of participants within the healthy and the C6 SCI groups is nearly twice of the C7 SCI group. However, it's necessary to take into account that five movement recordings have been analyzed for each person, and in this sense, the results obtained seemed to be sound.

On the other hand, the comparison with other indices in the literature is scarce due to there is no evidence of similar kinematic indices, designed for quantifying UL dexterity and ability during the execution of a complete ADL, whose computation is made from the combination of several kinematic variables. Moreover, the lack of standardization in the experimental protocols and the functional tasks chosen complicate the comparison between studies [38]. 
Above, in previous paragraphs, we have exposed that an advantage of this scoring system proposed is that may allow an easier understanding of the patient progress with respect to himself and to what extent his motor performance gets closer or away with respect to the reference pattern. However, in this paper, only the discriminative capability of the kinematic indices proposed has been proved with the aim of detecting and quantifying UL impairments. So, further research is needed, and the following step is to analyze the physiological meanings of the derived dimensionless indices for their possible use as evaluative measurements in a clinical setting. To reach that purpose, the validity, reliability, and the responsiveness to the change must be proved for each kinematic index [3]. The first step is to correlate the kinematic indices proposed with the clinical scales more frequently used in SCI patients [2, 41]; and then to analyze the responsiveness to the change [33], taking into account the minimal detectable change in each index [43]. These objectives should be addressed in a sample of patients with incomplete cervical SCI, taking into account that these patients present capabilities for improving the motor performance in relation to the UL functionality. For that reason, these objectives are beyond the scope of this research.

\section{Conclusions}

In this paper, three kinematic indices have been proposed with the aim of quantifying UL functional impairments in people with SCI. These indices, accuracy, efficiency, and coordination, are computed from the hand path and shoulder and elbow joint angles during the performance of the ADL of drinking. They are adequate for discriminating between healthy and people with different metameric level of cervical SCI. Further research is needed for analyzing the indices capability as evaluative measurements, including the analysis of their validity, reliability, and sensitivity to the change.

Acknowledgments The research for this manuscript has been partially funded by grant from the Spanish Ministry of Science and Innovation CONSOLIDER INGENIO, project HYPER (Hybrid NeuroProsthetic and neuroRobotic Devices for Functional Compensation and Rehabilitation of Motor Disorders, CSD 2009-00067).

\section{Compliance with ethical standards}

Conflict of interest Authors disclose any financial and personal relationships with other people or organizations that could inappropriately influence (bias) their work.

Ethical approval "All procedures performed in studies involving human participants were in accordance with the ethical standards of the institutional and local research committee and with the 1964
Helsinki Declaration and its later amendments or comparable ethical standards."

\section{References}

1. Aizawa J, Masuda T, Koyama T, Nakamaru K, Isozaki K, Okawa A, Morita S (2010) Three-dimensional motion of the upper extremity joints during various activities of daily living. J Biomech 43(15):2915-2922

2. Beninato M, O'Kane KS, Sullivan PE (2004) Relationship between motor FIM and muscle strength in lower cervical-level spinal cord injuries. Spinal Cord 42(9):533-540

3. Boyce WF, Gowland C, Rosenbaum PL, Lane M, Plews N, Goldsmith C, Zdrobov S (1991) Measuring quality of movement in cerebral palsy: a review of instruments. Phys Ther 71(11):813-819

4. Broeks JG, Lankhorst GJ, Rumping K, Prevo AJH (1999) The long-term outcome of arm function after stroke: results of a follow-up study. Disabil Rehabil 21(8):357-364

5. Butler EE, Ladd AL, LaMont LE, Rose J (2010) Temporal-spatial parameters of the upper limb during a reach \& grasp cycle for children. Gait posture 32(3):301-306

6. Cacho EWA, de OliveiraR Ortolan RL, Varoto R, Cliquet A (2011) Upper limb assessment in tetraplegia: clinical, functional and kinematic correlations. Int J Rehabil Res 34(1):65-72

7. Catz A, Tamir A, Itzkovich M (1998) SCIM- Spinal Cord Independence Measure: a new disability scale for patients with spinal cord lesions. Spinal Cord 36(734):735

8. Celik O, O'Malley MK, Boake C, Levin HS, Yozbatiran N, Reistetter T (2010) Normalized movement quality measures for therapeutic robots strongly correlate with clinical motor impairment measures. IEEE Trans Neural Sys Rehabil 18(4):433-444

9. Chang JJ, Wu TI, Wu WL, Su FC (2005) Kinematical measure for spastic reaching in children with cerebral palsy. Clin Biomech 20(4):381-388

10. Colombo R, Pisano F, Micera S, Mazzone A, Delconte C, Carrozza MC, Minuco G (2008) Assessing mechanisms of recovery during robot-aided neurorehabilitation of the upper limb. Neurorehabil Neural Repair 22(1):50-63

11. Colombo R, Sterpi I, Mazzone A, Delconte C, Pisano F (2012) Taking a lesson from patients' recovery strategies to optimize training during robot-aided rehabilitation. IEEE Trans Neural Sys Rehabil 20(3):276-285

12. Culmer PR, Levesley MC, Mon-Williams M, Williams JH (2009) A new tool for assessing human movement: the kinematic assessment tool. J Neurosci Method 184(1):184-192

13. de de los Reyes-Guzmán A, Dimbwadyo-Terrer I, TrincadoAlonso F, Monasterio-Huelin F, Torricelli D, Gil-Agudo A (2014) Quantitative assessment based on kinematic measures of functional impairments during upper extremity movements: a review. Clin Biomech 29(7):719-727

14. de los Reyes-Guzmán A, Gil-Agudo A, Peñasco-Martín B, SolísMozos M, del Ama-Espinosa A, Pérez-Rizo E (2010) Kinematic analysis of the daily activity of drinking from a glass in a population with cervical spinal cord injury. J Neuroeng Rehabil 7(1):41

15. Dipietro L, Krebs HI, Fasoli SE, Volpe BT, Stein J, Bever C, Hogan N (2007) Changing motor synergies in chronic stroke. J Neurophysiol 98(2):757-768

16. Jaspers E, Desloovere K, Bruyninckx H, Klingels K, Molenaers G, Aertbeliën E, Feys H (2011) Three-dimensional upper limb movement characteristics in children with hemiplegic cerebral palsy and typically developing children. Res Dev Disabil 32(6):2283-2294 
17. Jebsen RH, Taylor NEAL, Trieschmann RB, Trotter MJ, Howard LA (1969) An objective and standardized test of hand function. Arch Phys Med Rehabil 50(6):311

18. Kamper DG, McKenna-Cole AN, Kahn LE, Reinkensmeyer DJ (2002) Alterations in reaching after stroke and their relation to movement direction and impairment severity. Arch Phys Med Rehabil 83(5):702-707

19. Keith RA, Granger CV, Hamilton BB, Sherwin FS (1987) The functional independence measure. Adv Clin Rehabil 1:6-18

20. Kim K, Song WK, Lee J, Lee HY, Park DS, Ko BW, Kim J (2014) Kinematic analysis of upper extremity movement during drinking in hemiplegic subjects. Clin Biomech 29(3):248-256

21. Klotz MCM, Kost L, Braatz F, Ewerbeck V, Heitzmann D, Gantz S, Wolf SI (2013) Motion capture of the upper extremity during activities of daily living in patients with spastic hemiplegic cerebral palsy. Gait Posture 38(1):148-152

22. Lang CE, Wagner JM, Bastian AJ, Hu Q, Edwards DF, Sahrmann SA, Dromerick AW (2005) Deficits in grasp versus reach during acute hemiparesis. Exp Brain Res 166(1):126-136

23. Lang CE, Wagner JM, Edwards DF, Sahrmann SA, Dromerick AW (2006) Recovery of grasp versus reach in people with hemiparesis poststroke. Neurorehabil Neural Rep 20(4):444-454

24. Lum PS, Mulroy S, Amdur RL, Requejo P, PrilutskyBI Dromerick AW (2009) Gains in upper extremity function after stroke via recovery or compensation: potential differential effects on amount of real-world limb use. Top Stroke Rehabil 16(4):237-253

25. Magermans DJ, Chadwick EKJ, Veeger HEJ, Van Der Helm FCT (2005) Requirements for upper extremity motions during activities of daily living. Clin Biomech 20(6):591-599

26. Mahoney FI (1965) Functional evaluation: the Barthel index. Maryland State Med J 14:61-65

27. Mateo S, Revol P, Fourtassi M, Rossetti Y, Collet C, Rode G (2013) Kinematic characteristics of tenodesis grasp in C6 quadriplegia. Spinal cord 51(2):144-149

28. Maynard FM, Bracken MB, Creasey GJFD, Ditunno JF, Donovan WH, Ducker TB, Young W (1997) International standards for neurological and functional classification of spinal cord injury. Spinal Cord 35(5):266-274

29. Merlo A, Longhi M, Giannotti E, Prati P, Giacobbi M, Ruscelli E, Mazzoli D (2012) Upper limb evaluation with robotic exoskeleton. Normative values for indices of accuracy, speed and smoothness. NeuroRehabilitation 33(4):523-530

30. Murgia A, Kyberd P, Barnhill T (2010) The use of kinematic and parametric information to highlight lack of movement and compensation in the upper extremities during activities of daily living. Gait Posture 31(3):300-306

31. Murphy MA, Sunnerhagen KS, Johnels B, Willén C (2006) Three-dimensional kinematic motion analysis of a daily activity drinking from a glass: a pilot study. J Neuroeng Rehabil 3(1):18

32. Murphy MA, Willén C, Sunnerhagen KS (2011) Kinematic variables quantifying upper-extremity performance after stroke during reaching and drinking from a glass. Neurorehabil Neural Rep 25(1):71-80

33. Murphy MA, Willén C, Sunnerhagen KS (2013) Responsiveness of upper extremity kinematic measures and clinical improvement during the first three months after stroke. Neurorehab Neural Rep 27(9):844-853

34. Osu R, Ota K, Fujiwara T, Otaka Y, Kawato M, Liu M (2011) Quantifying the quality of hand movement in stroke patients through three-dimensional curvature. J Neuroeng Rehabil 8(1):62

35. Petuskey K, Bagley A, Abdala E, James MA, Rab G (2007) Upper extremity kinematics during functional activities: threedimensional studies in a normal pediatric population. Gait Posture 25(4):573-579

36. Rohrer B, Fasoli S, Krebs HI, Hughes R, Volpe B, Frontera WR, Hogan N (2002) Movement smoothness changes during stroke recovery. J Neurosci 22(18):8297-8304
37. Thies SB, Tresadern PA, Kenney LP, Smith J, Howard D, Goulermas JY, Rigby J (2009) Movement variability in stroke patients and controls performing two upper limb functional tasks: a new assessment methodology. J Neuroeng Rehabil 6:2

38. van Andel CJ, Wolterbeek N, Doorenbosch CA, Veeger DH, Harlaar J (2008) Complete 3D kinematics of upper extremity functional tasks. Gait Posture 27(1):120-127

39. Van den Berg MEL, Castellote JM, Mahillo-Fernandez I, de Pedro-Cuesta J (2010) Incidence of spinal cord injury worldwide: a systematic review. Neuro Epidemiol 34(3):184-192

40. Van Der Heide JC, Fock JM, Otten B, Stremmelaar E, HaddersAlgra M (2005) Kinematic characteristics of reaching movements in preterm children with cerebral palsy. Pediatr Res 57(6):883-889

41. Van Tuijl JH, Janssen-Potten YJ, Seelen HA (2002) Evaluation of upper extremity motor function tests in tetraplegics. Spinal Cord 40(2):51-64

42. Vergaro E, Casadio M, Squeri V, Giannoni P, Morasso P, Sanguineti V (2010) Self-adaptive robot training of stroke survivors for continuous tracking movements. J Neuroeng Rehabil 7(1):1

43. Wagner JM, Rhodes JA, Patten C (2008) Reproducibility and minimal detectable change of three-dimensional kinematic analysis of reaching tasks in people with hemiparesis after stroke. Phys Ther 88(5):652-663

44. Wyndaele M, Wyndaele JJ (2006) Incidence, prevalence and epidemiology of spinal cord injury: what learns a worldwide literature survey? Spinal Cord 44(9):523-529

45. Yang N, Zhang M, Huang C, Jin D (2002) Motion quality evaluation of upper limb target-reaching movements. Med Eng Phys 24(2): $115-120$

46. Zhou $\mathrm{H}, \mathrm{Hu} \mathrm{H}$ (2008) Human motion tracking for rehabilitation-A survey. Biomed Signal Proces 3(1):1-18 